\title{
Application of Ab Initio Results in Modeling Phase Diagrams Containing Complex Phases*
}

\author{
Mojmír ŠOB**,***,****, Aleš KROUPA****, Jana PAVLU゚**,***,****

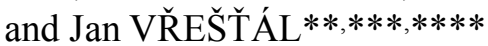 \\ ** Central European Institute of Technology, CEITEC MU, Masaryk University, Brno, Czech Republic \\ E-mail: sob@chemi.muni.cz \\ *** Department of Chemistry, Faculty of Science, Masaryk University, Brno, Czech Republic \\ **** Institute of Physics of Materials, Academy of Sciences of the Czech Republic, Brno, Czech Republic
}

\begin{abstract}
$\mathrm{Ab}$ initio electronic structure theory has achieved considerable reliability concerning predictions of physical and chemical properties and phenomena. It provides understanding of matter at the atomic and electronic scale with an unprecedented level of details and accuracy. In the present contribution, the electronic structure theory and state-of-the-art ab initio calculation methods in solids are briefly reviewed and the application of the calculated total energy differences between various phases (lattice stabilities) is illustrated on construction of phase diagrams by the CALPHAD (CALculation of PHAse Diagrams) method in systems containing phases with complex structures, as e.g. sigma phase. Particular examples include description of the sigma phase in the $\mathrm{Fe}-\mathrm{Cr}$ system and prediction of the phase composition of super-austenitic steels. It is shown that the utilization of $\mathrm{ab}$ initio results introduces a solid basis of the energetics of systems with complex phases, allows to avoid unreliable estimates and extrapolations of Gibbs energies and brings more physics into the CALPHAD method.
\end{abstract}

Key words: Computer Aided Analysis, Iron and Steel, Phase Transformations, First-Principles Analysis

\section{Introduction}

The combination of ab initio calculations and CALPHAD (CALculation of PHAse Diagrams) method ${ }^{(1)(2)}$ is very efficient and powerful approach, especially when applied to systems with complex phases, where we do not have enough data from experiment and have to resort to first-principles calculations to be able to construct a reliable Gibbs energy of such a phase. This approach is becoming increasingly popular recently, mainly in connection with a steady increase in the computing power and development of more and more precise approximations for the solution of quantum mechanical equations.

\section{Concepts and methodology of the CALPHAD method}

The CALPHAD method is a semi-empirical approach ${ }^{(1)(2)}$, allowing the modeling of thermodynamic properties of multicomponent systems and consequent calculation of corresponding phase diagrams. Its main feature is the combination of experimental observation and theoretical modeling and it is significantly dependent on the amount and quality of experimental data available. Exactly here is the place for application of the ab initio calculations, as very often we do not have enough experimental input for a reliable 
description of all phases present in the system and we may perform "a measurement in a computer", calculating the quantities needed from the first principles.

The CALPHAD method is based on a sequential modeling of multicomponent systems. We start with the assessment of Gibbs energy functions for pure elemental constituents and continue with the modeling of more complex phases such as binary and ternary solutions and intermetallic phases. This procedure transfers the problem of extrapolation of phase data to the problem of extrapolation of thermodynamic functions, which may be solved more precisely. Thermodynamic properties of a binary system can be calculated using the Gibbs energy expressions comprising contributions for both elemental constituents in each of the phases existing in the system. Further parameters are introduced to describe the mutual interaction between the constituents in each phase. It is possible to model such a system with a high precision if reliable experimental data are available. We need both the thermodynamic data describing the relevant properties of the phases (e.g. specific heats, enthalpies of formation or mixing, activities etc.; here we can include also the ab initio calculated energies of formation at the temperature of $0 \mathrm{~K}$ ) and adequate information on phase equilibria (e.g. invariant temperatures, equilibrium compositions and concentration of phases). By combining robust thermodynamic descriptions of binary and ternary alloy systems, it is possible to predict phase equilibria also in higher-order systems. Deviations of theoretical predictions from the actual behavior of the system can be minimized by introducing additional parameters, but they must not influence the modeled thermodynamic properties and calculated phase diagrams of the lower-order systems.

The basic mathematical method used for the calculation of phase equilibria is a constrained minimization of Gibbs energy for a given temperature, pressure and overall composition. This approach is common to all currently available software packages for the modeling of thermodynamic properties and phase diagrams of multicomponent systems.

The total Gibbs energy of the system can be written as

$$
G^{\mathrm{tot}}=\sum_{p} w^{\mathrm{p}} G_{\mathrm{m}}{ }^{\mathrm{p}} .
$$

Here $w^{\mathrm{p}}$ is the amount of the phase $\mathrm{p}$ in the system and the molar Gibbs energy of any phase, $G_{\mathrm{m}}^{\mathrm{p}}$, is expressed as

$$
G_{\mathrm{m}}^{\mathrm{p}}=G_{\mathrm{ref}}^{\mathrm{p}}+G_{\mathrm{id}}^{\mathrm{p}}+G_{\mathrm{E}}^{\mathrm{p}}+G_{\mathrm{mag}}^{\mathrm{p}}+G_{\mathrm{press}}^{\mathrm{p}}+\ldots
$$

where

$$
G_{\text {ref }}{ }^{\mathrm{p}}=\sum_{i} x_{i}{ }^{0} G_{i}^{\mathrm{p}}
$$

is the reference level of the Gibbs energy. It is calculated as a weighted sum of the Gibbs energies ${ }^{0} G_{\mathrm{i}}{ }^{\mathrm{p}}$ of the constituents $i$ in the crystallographic structure corresponding to the phase $\mathrm{p}$ related to a selected reference state; $x_{i}$ is the concentration of the constituent $i$ in the phase $\mathrm{p}$ (molar fraction). Typically the Gibbs energy of so-called Standard Element Reference (SER) state is selected as this reference, which is the Gibbs energy of the constituent $i$ in the stable crystallographic structure, existing at room temperature of 298.15 $\mathrm{K}$ and standard pressure of 1 bar (e.g. fcc $\mathrm{Cu}$, bcc Mo or bcc ferromagnetic $\mathrm{Fe}$ ).

\section{Ab initio input to CALPHAD calculations}

$\mathrm{Ab}$ initio (or first-principles) electronic structure calculations in solids are based on 
fundamental quantum theory. Such calculations are routinely performed within the framework of density functional theory (DFT) in which the complicated many-body motion of all electrons is replaced by an equivalent but simpler problem of a single electron moving in an effective potential.

State-of-the-art electronic structure calculations provide an understanding of matter at the atomic and electronic scale with an unprecedented level of detail and accuracy. In many cases, we are able not only to simulate experiment but also to design new molecules and materials and to predict their properties before actually synthesizing them. A computational simulation can also provide data on the atomic scale that are inaccessible experimentally. In contrast to semi-empirical approaches (where the adjustable parameters are fitted to the properties of the ground state structure and, therefore, may not be transferable to non-equilibrium configurations), ab initio calculations, depending on the quality of approximation, are reliable far from the equilibrium as well. In connection with the CALPHAD method, they provide total energies (lattice stabilities) of the elemental constituents of the system studied and their vibrational entropies.

The Gibbs energy ${ }^{0} G_{i}^{\mathrm{p}}$ of the constituent $i$ in the phase p may be written in the form

$$
{ }^{0} G_{i}^{\mathrm{p}}={ }^{0} G_{i}^{\mathrm{SER}}+\left(H_{i}^{\mathrm{p}}-H_{i}^{\mathrm{SER}}\right)-T\left(S_{i}^{\mathrm{p}}-S_{i}^{\mathrm{SER}}\right),
$$

where ${ }^{0} G_{i}$ SER is Gibbs energy of component $i$ in the SER structure, $\Delta^{0} H_{i}^{\mathrm{p} \text {-SER }}=H_{i}^{\mathrm{p}}-H_{i}{ }^{\mathrm{SER}}$ is the enthalpy difference between the phase $\mathrm{p}$ and SER structure and $\Delta^{0} S_{i}^{\mathrm{p} \text {-SER }}=S_{i}^{\mathrm{p}}-S_{i}^{\mathrm{SER}}$ is the corresponding entropy difference.

At $T=0 \mathrm{~K}$ we may write that $\Delta^{0} H_{i}{ }^{\mathrm{p} \text {-SER }}(T=0)=\Delta^{0} E_{i}{ }^{\mathrm{p}-\mathrm{SER}}(T=0)$, i.e. the difference in enthalpies is equal to the difference of total energies because the total energies at $0 \mathrm{~K}$ are calculated at equilibrium volumes where it holds $(\partial U / \partial V)_{T}=T(\partial p / \partial T)_{V}-p=0(U$ is the internal energy and $p$ is the pressure here). The difference in enthalpies, $\Delta^{0} H_{i}^{\mathrm{p} \text {-SER }}$ at finite temperature, is then obtained, according to the Kirchhoff's law, as

$$
\Delta^{0} H_{i}^{\mathrm{p} \text {-SER }}=\Delta^{0} E_{i}^{\mathrm{p}-\mathrm{SER}}+\int \Delta C_{\mathrm{p}}^{\mathrm{p}-\mathrm{SER}} \mathrm{d} T
$$

where $C_{\mathrm{p}}$ is the heat capacity at constant pressure (the subscript $i$ is dropped here). In the region without a phase transformation, the vibrational contribution to the entropy can be expressed by

$$
\Delta^{0} S_{i}^{\mathrm{p}-\mathrm{SER}}=\int\left(\Delta C_{\mathrm{p}}^{\mathrm{p}-\mathrm{SER}} / T\right) \mathrm{d} T
$$

At the temperature of $0 \mathrm{~K}$ and at the equilibrium structures of the phases studied, the differences in enthalpies between the phase $\mathrm{p}$ and the SER state are equal to the total energy differences $\left(\Delta^{0} H^{\mathrm{p} \text {-SER }}=\Delta^{0} E^{\mathrm{p} \text {-SER }}\right)$. These can be calculated ab initio. Approximating the $\Delta C_{\mathrm{p}}{ }^{\mathrm{p} \text {-SER }}$ by a simple relation $\Delta C_{\mathrm{p}}{ }^{\mathrm{p} \text {-SER }}=a+b T$, we obtain, by integration in Eqs. (2) and (3) and substitution into Eq. (1), the expression

$$
\Delta^{0} G_{i}^{\mathrm{p} \text {-SER }}=\Delta^{0} E_{i}^{\mathrm{p} \text {-SER }}+T(a-b-a \ln T)+(b / 2) T^{2}
$$

and, assuming $b=0$ for metallic systems and for not very extended temperature interval, we may write

$$
\Delta^{0} G_{i}^{\mathrm{p} \text {-SER }}=\Delta^{0} E_{i}^{\mathrm{p}-\mathrm{SER}}+\left(\Delta C_{\mathrm{p}}^{\mathrm{p}-\mathrm{SER}}\right) T-\left(\Delta C_{\mathrm{p}}^{\mathrm{p}-\mathrm{SER}}\right) T \ln T .
$$


This equation may be successfully employed in the phase diagram calculations. Usually, $\Delta^{0} E_{i}^{\mathrm{p} \text {-SER }}$ is calculated ab initio and $\Delta C_{\mathrm{p}}^{\mathrm{p} \text {-SER }}$ is fitted to the experimental phase equilibrium data, as we have done that e.g. in papers ${ }^{(3)-(5)}$. However, also the value of $\Delta C_{\mathrm{p}}{ }^{\mathrm{p} \text {-SER }}$ may be taken from ab initio or other calculations (e.g. from phonon spectra ${ }^{(6)-(8)}$ ) or from other measurements (e.g. from the analysis of Einstein or Debye functions). In this way, we could further reduce the number of fitting parameters needed for description of phase diagrams.

At present, there are a lot of ab initio electronic structure methods. They may be distinguished according to the choice of the basis functions used for the expansion of the electronic wavefunctions. The better we choose them (according to the character of the problem), the smaller is the number needed. One uses augmented (APW) or orthogonalized (OPW) plane waves, linear muffin-tin orbitals (LMTO), linear combination of atomic (LCAO), Gaussian (LCGO) and augmented Slater-type (LASTO) orbitals, augmented spherical waves (ASW), etc. The Korringa-Kohn-Rostoker (KKR) method proceeds by the use of the Green function of the Kohn-Sham equation and is also called the Green function (GF) method. The pseudopotential approach is also widely used. A detailed description of these methods may be found in many books and articles (see e.g. ${ }^{(9)(10)}$ and references therein). Application of ab initio results to construction of phase diagrams by CALPHAD method and to predicting the crystal structures of materials may be found e.g. in Refs. ${ }^{(11)-(14)}$.

When performing ab initio electronic structure calculations, a proper description of the exchange-correlation energy, which is inherent to DFT, must be adopted. In many cases, local-density approximation (LDA) is sufficient. Here we suppose that the electron density can be treated locally as a uniform electron gas. The exchange-correlation energy at each point in the system is then calculated as that of a uniform electron gas of the same density. In ferro- and antiferromagnetic $3 \mathrm{~d}$ metals, such as $\mathrm{Cr}, \mathrm{Mn}, \mathrm{Fe}, \mathrm{Co}$ and $\mathrm{Ni}$, this approximation does not reproduce a correct energy order of crystal structures and a more sophisticated generalized gradient approximation (GGA) must be employed, which also includes gradient of the electron density ${ }^{(15)}$. In materials with narrow $4 \mathrm{f}$ and $5 \mathrm{f}$ bands or with other strongly correlated electrons, the so-called LDA+U approximation is used that includes the effect of a strong on-site Coulomb repulsion ${ }^{(16)}$. Also, hybrid exchange-correlation energy functionals are employed in these cases ${ }^{(17)}$.

In any particular study, one should choose a method which fits best to the properties of the systems investigated. For example, in most of our ab initio electronic structure calculations for systems containing $3 \mathrm{~d}$ metals, we employ the pseudopotential method implemented in the Vienna Ab initio Simulation Package (VASP) ${ }^{(18)-(20)}$. This code meets all our requirements regarding the computational speed and accuracy. We use projector augmented waves (PAW) pseudopotentials ${ }^{(21)}$ with exchange-correlation functional as parameterized by Perdew-Burke-Ernzerhof $(\mathrm{PBE}){ }^{(15)}$. This approach reproduces very well the energy order and lattice stabilities of the bcc, fcc and hep structures in Fe, Co and Ni and is very convenient for investigations of magnetic systems.

\section{Illustrative examples of application of ab initio results in CALPHAD calculations}

Binary sigma phases. Occurrence of the sigma phase in alloys is very unfavorable as it is brittle and therefore it causes a strong degradation of material (crack nucleation sites). In practice, it develops in heat-affected zones of welded superaustenitic stainless steels and it was concluded that it is formed after longer ageing times in the temperature range of $500-1100{ }^{\circ} \mathrm{C}$. It is also known that high concentrations of $\mathrm{Cr}$ and Mo promote precipitation of this phase. From the thermodynamic point of view, the sigma phase is very stable and its 
stability may be theoretically assessed using the Gibbs energy difference between the sigma phase and standard element reference (SER) states.

The crystal structure of sigma phase is tetragonal and its prototype is the FeCr sigma phase. It has the space group No. 136 (P42/mnm) and its repeat cell contains thirty atoms accommodated in five crystallographically inequivalent sublattices $2 \mathrm{a}, 4 \mathrm{f}, 8 \mathrm{i}, 8 \mathrm{i}$ ' and $8 \mathrm{j}$ (Fig. 1).

Table 1 The values of the total energy differences between the sigma phase and SER structures, calculated for $\mathrm{Cr}, \mathrm{Fe}$, and Mo by means of the VASP code ${ }^{(18)-(20)}$ combined with the PAW-PBE pseudopotential ${ }^{(21)(15)}$ at equilibrium structure parameters. Here AFM, FM and NM stand for antiferromagnetic, ferromagnetic and nonmagnetic state.

\begin{tabular}{|c|c|c|}
\hline Element & SER & $\Delta E^{\sigma-S E R}\left(\mathrm{~kJ} \mathrm{~mol}^{-1}\right)$ \\
\hline $\mathrm{Cr}$ & AFM bcc & 13.39 \\
\hline $\mathrm{Fe}$ & FM bcc & 25.78 \\
\hline $\mathrm{Mo}$ & NM bcc & 16.15 \\
\hline
\end{tabular}

The values of the total energy differences between the sigma phase and the SER structures, calculated for $\mathrm{Cr}, \mathrm{Fe}$ and Mo using the VASP code ${ }^{(18)-(20)}$ at equilibrium volume, including relaxation with respect to volume and c/a ratio, are summarized in Table 1 . They may be used for CALPHAD modeling of phase diagrams.
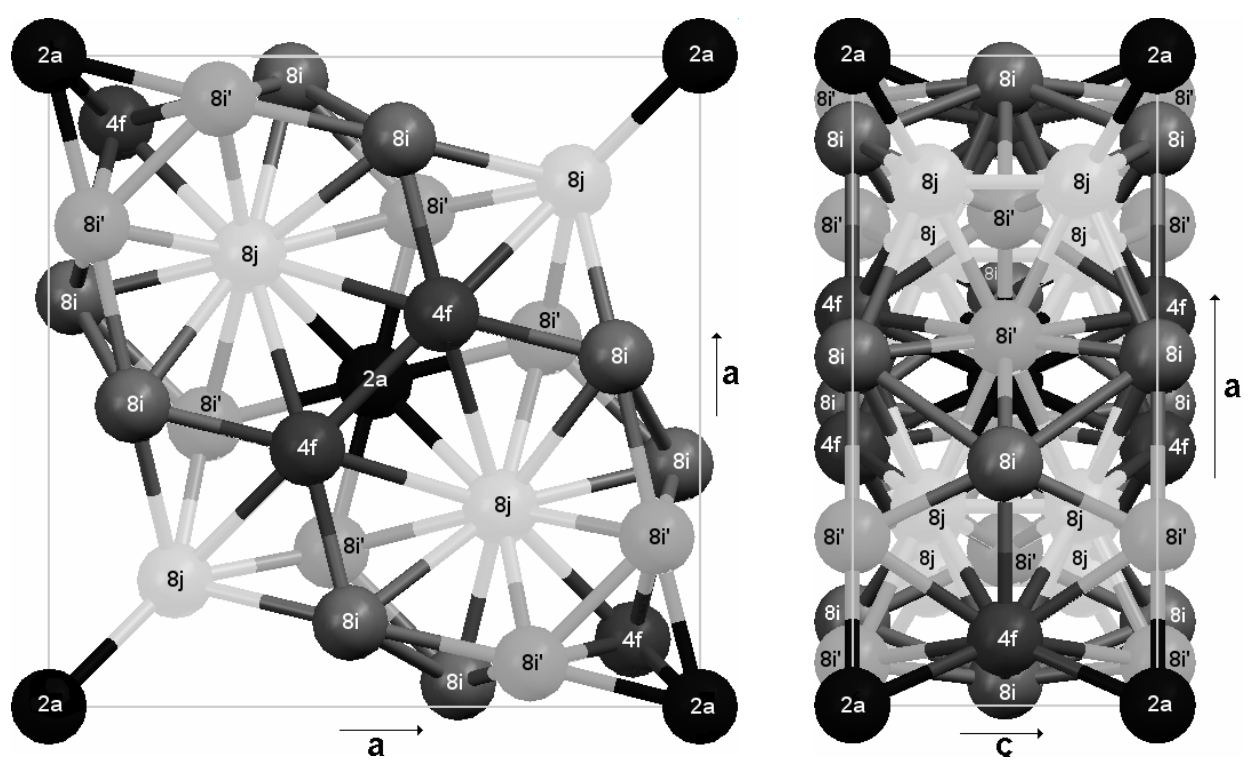

Fig. 1 The structure of the sigma phase. The brightness of atoms in sublattices is increasing in the order of positions $2 \mathrm{a}, 4 \mathrm{f}, 8 \mathrm{i}, 8 \mathrm{i}$ ' and $8 \mathrm{j}$. 


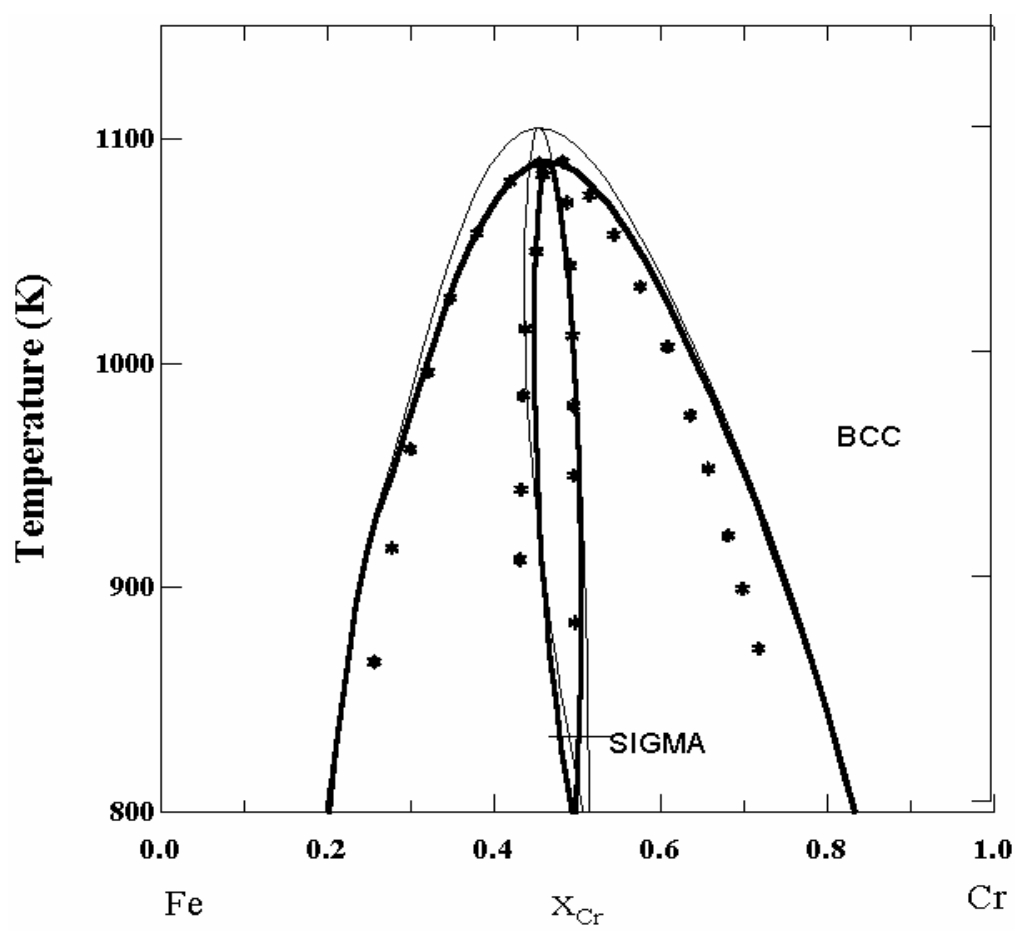

Fig. 2 Comparison of phase diagrams of $\mathrm{Cr}-\mathrm{Fe}{ }^{(3)}$. Thick line was obtained by a new two-sublattice model ${ }^{(22)}$, thin line by three-sublattice model (the data were taken from ${ }^{(23)}$ ). Asterisks represent the experimental results from ${ }^{(24)}$.

Fig. 2 shows two phase diagrams of Fe-Cr system calculated using two different models in the region of existence of the sigma phase. It may be seen that our new two-sublattice model ${ }^{(13)}$ incorporating ab initio results exhibits a better agreement with experimental data ${ }^{(24)}$ than the old three-sublattice approach (not employing ab initio results).

Let us note that inclusion of magnetic ordering in the $\mathrm{Cr}-\mathrm{Fe}$ sigma phase calculations causes the decrease of energy of formation and results in the shift of stability region towards the configurations with a higher concentration of iron ${ }^{(5)}$. We predict that $\mathrm{Cr}-\mathrm{Fe}$ (and also $\mathrm{Cr}-\mathrm{Co}$ ) sigma phases are magnetically ordered at $0 \mathrm{~K}$, which corresponds to experimental findings. The largest part of magnetization is carried by the iron (or cobalt) atoms and chromium atoms do not contribute to this effect very much although they induce a decrease of the total magnetic moment by their antiferromagnetic behaviour at some sublattices.

Modern high-Cr and super-austenitic steels. Empirically and theoretically correct and robust assessments of binary and ternary systems will allow us to use the CALPHAD method for the modeling of phase equilibria and thermodynamic functions in multicomponent systems, important for industrial and scientific applications. The thermodynamic data of lower-order systems are usually collected in thermodynamic databases, which can be general (covering broad range of elements) or specialized for specific purposes (e.g. Ni-based alloys, steels, solders). There are strict rules concerning the construction of such databases to keep the internal consistency of the data and allowing correct calculations. Such rules are described in general manner e.g. in ${ }^{(25)}$.

Some of the authors of the present paper developed a consistent database STEEL16 specialized for Fe-rich materials (mainly steels). This database contains 17 elements $(\mathrm{Fe}, \mathrm{Al}$, $\mathrm{C}, \mathrm{Co}, \mathrm{Cr}, \mathrm{Cu}, \mathrm{Mn}, \mathrm{Mo}, \mathrm{N}, \mathrm{Nb}, \mathrm{Ni}, \mathrm{Si}, \mathrm{Ta}, \mathrm{Ti}, \mathrm{V}, \mathrm{W}, \mathrm{Zr}$ ), important for the systems corresponding to commonly used steels. The binary and ternary assessments of relevant 
systems both from literature and from their own work ${ }^{(26)}$ are included. Some of these assessments use the above-described approach combining the semi-empirical and ab initio methods.

Here we illustrate application of the CALPHAD modelling and the STEEL16 database for prediction of the phase equilibria in the super-austenitic NICROFER steels (see Table 2). We used the database STEEL16 for the calculation of phase equilibria in steels. Verification of the prediction of phase equilibria in these steels has been performed by applying a long-term annealing. The experimental results obtained by the EDX analysis for samples of Nicrofer 3033 and Nicrofer 3127 following annealing at $700^{\circ} \mathrm{C}$ for $6000 \mathrm{hrs}$ (27) were compared with calculated phase composition in Tables 3 and 4.

Good qualitative agreement between the calculations performed using the STEEL16 database and the experimentally determined phase compositions ${ }^{(28)}$ in the samples is evident.

Table 2 Chemical composition of the samples of Nicrofer 3033 and Nicrofer 3127 steels.

\begin{tabular}{|c|c|c|c|c|c|c|c|c|c|c|c|}
\hline Steel (wt.\%) & $\mathrm{Cr}$ & $\mathrm{Ni}$ & $\mathrm{Mn}$ & $\mathrm{Si}$ & $\mathrm{Mo}$ & $\mathrm{Cu}$ & $\mathrm{S}$ & $\mathrm{P}$ & $\mathrm{C}$ & $\mathrm{N}$ & $\mathrm{Fe}$ \\
\hline Nicrofer 3033 & 32.8 & 30.9 & 0.64 & 0.31 & 1.67 & 0.58 & 0.004 & 0.010 & 0.007 & 0.39 & bal. \\
Nicrofer 3127 & 27.0 & 31.0 & 1.6 & 0.30 & 6.4 & 1.3 & $\mathrm{a}$ & $\mathrm{a}$ & 0.009 & 0.20 & bal. \\
\hline
\end{tabular}

a - not determined

Table 3 Phases found experimentally after annealing at $700{ }^{\circ} \mathrm{C} / 6170 \mathrm{hrs}$ in Nicrofer 3033 and comparison with calculated equilibrium phase composition.

\begin{tabular}{|l|c|c|c|c|c|c|}
\hline & fcc (austenite) & bcc (Cr-rich) & $\sigma$ phase & $\pi$ phase & $\mathrm{M}_{23} \mathrm{C}_{6}$ & $\mathrm{M}_{2}(\mathrm{C}, \mathrm{N})(\mathrm{hcp})$ \\
\hline Experiment & + & + & $9.0 \%$ & + & 0 & 0 \\
\hline Calculation & $77.0 \%$ & $13.9 \%$ & $4.4 \%$ & 0 & 0 & $4.7 \%$ \\
\hline
\end{tabular}

+ found, 0 not found

Table 4 Phases found experimentally after annealing at $700{ }^{\circ} \mathrm{C} / 6000 \mathrm{hrs}$ in Nicrofer 3127 and calculated equilibrium phase composition.

\begin{tabular}{|l|c|c|c|c|c|c|c|}
\hline & $\begin{array}{c}\text { fcc } \\
\text { (austenite) }\end{array}$ & $\begin{array}{c}\text { bcc } \\
(\text { Cr-rich) }\end{array}$ & $\begin{array}{c}\sigma \\
\text { phase }\end{array}$ & $\begin{array}{c}\pi \\
\text { phase }\end{array}$ & $\mathrm{M}_{23} \mathrm{C}_{6}$ & $\mathrm{M}_{2}(\mathrm{C}, \mathrm{N})$ (hcp) & Laves \\
\hline Experiment & + & 0 & $38.0 \%$ & 0 & 0 & + & 0 \\
\hline Calculation & $72.9 \%$ & $6.0 \%$ & $18.6 \%$ & 0 & 0 & $2.5 \%$ & 0 \\
\hline
\end{tabular}

+ found, 0 not found

\section{Problems and challenges in CALPHAD modeling}

As it has been discussed above, CALPHAD modelling requires the expressions for Gibbs energies of all elemental constituents in all phase structures occurring in the system. If the system contains complex phases, no experimental data on structures of the constituents in these phases exist, however, the total energy differences with respect to some standard states at the temperature of $0 \mathrm{~K}$ (lattice stabilities) may be calculated ab initio.

In the case of complex intermetallic phases with complicated crystallographic structure we have another problem how to choose an appropriate model. If we decide to describe the studied phases with the help of a complicated formula, we can properly reflect the crystallographic situation but we also get a lot of degrees of freedom in the CALPHAD modeling that are difficult to handle. Sometimes the poorly estimated parameters can even 
cause problems in assessment of higher-order systems. Possible deficiencies in structural energy differences (lattice stabilities) may be easily compensated by entropy terms and interaction parameters (see e.g. Redlich-Kister polynomial for excess Gibbs energy (Eqs. 20,23 and 27 in $\left.^{(13)}\right)$. One can say that it is this great freedom, which allows CALPHAD to work so nicely. But it is also a disadvantage: some parameters can be hardly interpreted in physical terms and, therefore, wicked tongues state that the CALPHAD method is just a fitting scheme. We do not share this opinion. There is no doubt about good-quality description of phase diagrams of many systems and the commercially available software (ThermoCalc, MTDATA, FactSage) using the CALPHAD approach and consistent thermodynamic databases have a great economical success. However, we do agree with some calls for more physics to CALPHAD. Inclusion of lattice stabilities calculated ab initio is one of the steps in this direction.

\section{Conclusions}

In the present contribution, we have shortly reviewed ab initio electronic structure calculations and the CALPHAD method and have shown that their combination gives a better physical insight into the construction of phase diagrams.

In view of approximations used, we cannot expect to be able to describe all details of complex phase diagrams now. However, the qualitative trends and general features are reproduced correctly by present calculations. Inclusion of ab initio results puts the energetics of the systems with complex phases on a solid basis, avoids unreliable estimates and extrapolations of Gibbs energies and brings more physics to the CALPHAD method.

\section{Acknowledgements}

This research was supported by the Research Projects AV0Z20410507 and MSM0021622410, by the Czech Science Foundation (Project No. P108/10/1908), by the Ministry of Education of Czech Republic (Projects No. OC10008 and LD11024) and by the Project CEITEC - Central European Institute of Technology (CZ.1.05/1.1.00/02.0068) from the European Regional Development Fund. The access to the MetaCentrum computing facilities (Project No. LM2010005) is highly appreciated. The phase diagram calculations were performed using ThermoCalc software.

\section{References}

(1) Saunders, N. and Miodownik, A.P., CALPHAD (A Comprehensive Guide), (1998), Elsevier.

(2) Lukas, H.L., Fries, S.G. and Sundman, B., Computational Thermodynamics, (2007), Cambridge University Press.

(3) Houserová, J., Friák, M., Šob, M. and Vřešt’ál, J., Comput. Mater. Sci., Vol. 25 (2002), p. 562.

(4) Pavlů, J., Vřešt’ál, J. and Šob, M., CALPHAD, Vol. 34 (2009), p. 215.

(5) Pavlů, J., Vřešt'ál, J. and Šob, M., Intermetallics, Vol. 18 (2010), p. 212.

(6) Ottonello, G., Zuccolini, M.V. and B. Civalleri, B., CALPHAD, Vol. 33 (2009), p. 457.

(7) Dubiel, S.M., Cieslak, J., Sturhahn, W., Sternik, M., Piekarz, P., Stankov, S. and Parlinski, K., Phys. Rev. Lett., Vol. 104 (2010), p. 155503.

(8) Fultz, B., Progr. Mat. Sci., Vol. 55 (2010), 247.

(9) Turek, I., Drchal, V., Kudrnovský, J., Šob, M. and Weinberger, P., Electronic Structure of Disordered Alloys, Surfaces and Interfaces, (1997), Kluwer.

(10) Martin, R.M., Electronic Structure. Basic Theory and Practical Methods, (2004), Cambridge University Press. 
(11) Wang, Y., Curtarolo, S., Jiang, C., Arroyave, R., Wang, T., Ceder, G., Chen, L.-Q. and Liu, Z.-K., CALPHAD, Vol. 28 (2004), p. 79.

(12) Curtarolo, S., Morgan, D. and Ceder, G., CALPHAD, Vol. 29 (2005), p. 163.

(13) Šob, M., Kroupa, A., Pavlů, J. and Vřešt’ál, J., Solid State Phenomena Vol. 150 (2009), p. 1.

(14) Liu, Z.-K., J. Phase Equilib. Diff., Vol. 30 (2009), p. 517.

(15) Perdew, J.P., Burke, K. and Ernzerhof, M., Phys. Rev. Lett., Vol. 77 (1996), p. 3865.

(16) Liechtenstein, A.I., Anisimov, V.I. and Zaanen, J., Phys. Rev. B, Vol. 52 (1995), p. R5467.

(17) Tran, F., Blaha, P., Schwarz, K. and Novák, P., Phys. Rev. B, Vol. 74 (2006), p. 155108.

(18) Kresse, G. and Furthmüller, J: Comput. Mater. Sci., Vol. 6 (1996), p. 15; Kresse, G. and Furthmüller, J., Phys. Rev. B, Vol. 54 (1996), p. 11169.

(19) Hafner, J., Comp. Phys. Commun., Vol. 177 (2007), p. 6.

(20) Hafner, J., J. Comp. Chem., Vol. 29 (2008), p. 2044.

(21) Blöchl, P., Phys. Rev. B, Vol. 50 (1994), p. 17953; Kresse, G. and Joubert, J., Phys. Rev. B, Vol. 59 (1999), p. 1758.

(22) Vřešt’ál, J., Arch. Metall., Vol. 46 (2001), p. 239.

(23) Andersson, J.-O. and Sundman, B., CALPHAD, Vol. 11 (1987), p. 83.

(24) Cook, A.J. and Jones, F.W., J. Iron Steel Inst., Vol. 148 (1943), p. 217.

(25) Kroupa, A., Dinsdale, A.T., Watson, A., Vřeštál, J., Vízdal, J. and Zemanová, A., JOM, Vol. 59 (2007), p. 20.

(26) Kroupa, A., Havránková, J., Coufalová, M., Svoboda, M. and Vřešt’ál, J., J. Phase Equilib. Diff., Vol. 22 (2001), p. 312.

(27) Kraus, M., Kroupa, A., Miodownik, P., Svoboda, M. and Vřešt’ál, J., Int. J. Mat. Res., Vol. 101 (2010), p. 729.

(28) Vřešt’ál, J., Kroupa, A. and Šob, M., Comput. Mater. Sci., Vol. 38 (2006), p. 298. 